\title{
Die Rezension als studentische Textart zur Einübung von zentralen wissenschafts- sprachlichen Handlungen
}

\author{
Albrecht Klemm, Stefan Rahn, Renate Riedner
}

\begin{abstract}
Zusammenfassung
In diesem Artikel wird das Potential von wissenschaftlichen Rezensionen für die textsorten- und handlungsbezogene Wissenschaftssprachvermittlung aufgezeigt. Der Ausgangspunkt ist eine handlungsbezogene Bestimmung der Textart, wobei zwei komplexe Sprachhandlungen als zentral angesehen werden: das zusammenfassende Referieren und das Bewerten. Anhand von Belegen aus einem Korpus von 20 studentischen Rezensionen werden verschiedene Teilhandlungen beim zusammenfassenden Referieren und Bewerten sowie spezifische Merkmale und Problembereiche studentischer Rezensionen diskutiert. Dabei wird deutlich, dass wissenschaftliche Rezensionen aufgrund ihrer Handlungsspezifik eine wichtige Rolle als studentische Textart in der Wissenschaftssprachvermittlung spielen können.
\end{abstract}

\section{Einleitung}

Das Verfassen von wissenschaftlichen Texten in der Fremdsprache Deutsch stellt eine besondere Herausforderung für ausländische Studierende und Wissenschaftler ${ }^{1}$ dar. Um erfolgreich wissenschaftlich schreiben zu können, müssen sie:

- über ein hohes Sprachniveau in der Fremdsprache verfügen, welches sowohl das Beherrschen der allgemeinen bzw. »alltäglichen Wissenschaftssprache« (vgl. Ehlich 1993) als auch die Kenntnis fachspezifischer Begriffe und Wendungen einschließt,

1 Männliche Personenbezeichnungen im Text sind als generische Maskulina zu verstehen - sie schließen das weibliche Geschlecht prinzipiell mit ein. 
- das für die Erfüllung der Schreibaufgabe notwendige fachliche Wissen abrufen und der Kommunikationssituation angemessen versprachlichen,

- die für ihren Forschungsbereich relevanten wissenschaftlichen und studentischen Textarten (z. B. Artikel, Exzerpt, Mitschrift, Rezension, Exposé) und die daran geknüpften Erwartungen der Wissenschaftsgemeinschaft kennen,

- zentrale wissenschaft(ssprach)liche Handlungen wie das Argumentieren, das Zusammenfassen, Referieren und Bewerten von rezipiertem wissenschaftlichen Wissen in ihrer Funktion verstehen und selbst ausführen können sowie die Bedeutung dieser sprachlichen Handlungen für das diskursive deutsche Wissenschaftsverständnis (»Eristik«, vgl. Ehlich 1993) erkennen,

- über Schreibprozesswissen verfügen, d.h. ihr Schreiben durch den Einsatz von Schreibstrategien planen, durchführen und evaluieren können.

Ausländische Studierende beim Aufbau dieser wissenschaftssprachlichen Kompetenz zu unterstützen, ist ein wesentliches Ziel des Moduls »Deutsch als Wissenschaftssprache «, das am Herder-Institut der Universität Leipzig für internationale Studierende in binationalen Master-Studiengängen angeboten wird. Grundlegend für die Konzeption des Moduls ist die Annahme, dass der Aufbau sprachlicher Handlungsfähigkeit im Kontext der Wissenschafts- und Diskurstraditionen des Faches gesehen werden muss und nicht davon losgelöst werden kann. Daraus ergibt sich, dass im Modul ein integrativer Ansatz verfolgt wird, bei dem wissenschaftliche Text- und Diskursarten, Wissenschaftssprache und Konventionen wissenschaftlicher Kommunikation mithilfe von auf den unmittelbaren Studienzusammenhang bezogenen Aufgabenstellungen vermittelt und eingeübt werden. Alle Schreibaufgaben des Moduls weisen dementsprechend einen konkreten Bezug zur wissenschaftlichen Arbeit der Studierenden auf, d.h. die Studierenden werden im Sinne einer »sukkursiven Sprachvermittlung « (Ehlich/ Graefen 2001) gezielt bei der Erarbeitung studienrelevanter Leistungen und damit individuell in ihrem wissenschaft(ssprach)lichen Handeln unterstützt. Sprachliche Formen werden in einem solchen Lehrkontext in ihrem Zusammenhang mit dem Gegenstand und den mit ihm verbundenen Denk- und Argumentationsweisen thematisiert (vgl. auch das Konzept des sach- und fachbezogenen Sprachenlernens nach Fandrych 2007).

Dementsprechend werden im Modul verschiedene aufeinander aufbauende und in Bezug zu konkreten wissenschaftlichen Projekten der Studierenden stehende Textund Diskursarten trainiert. Durch das Anfertigen von kommentierten Bibliographien und Exposés sowie das Präsentieren des Forschungsstands zu ihrer Forschungsarbeit mithilfe eines wissenschaftlichen Posters erwerben die Studierenden schrittweise Expertise in ihrem Fachgebiet und entwickeln gleichzeitig ihre wissenschaftssprachliche Handlungsfähigkeit weiter. Zum Abschluss des Moduls verfassen sie eine wissenschaftliche Rezension, die eine Publikation aus ihrem For- 
schungsbereich zum Gegenstand hat. Sie bauen dabei auf den im Laufe des Moduls eingeübten Text-/Diskursarten und wissenschaftssprachlichen Handlungen auf. Die wissenschaftliche Rezension steht im Zentrum des vorliegenden Beitrags, in dem wir uns der Frage widmen wollen, worin das Potential gerade dieser Textart für eine handlungsbezogene Wissenschaftssprachvermittlung besteht.

\section{Forschungsstand und handlungsbezogene Bestimmung der Textart »Wissenschaftliche Rezension «}

Die wissenschaftliche Rezension ist eine Textart, die auf einer kritischen Auseinandersetzung mit einer wissenschaftlichen Publikation, mit ihren Inhalten, Fragestellungen, Methoden, Daten usw. basiert. Sie ist in diesem Sinne eine textverarbeitende Textart mit Bezug auf einen Primärtext (vgl. Ehlich 1981). Die kritische Auseinandersetzung mit diesem Primärtext leistet zunächst der Rezensent, in der Regel ein Wissenschaftler mit themenspezifischer Expertise, indem er die jeweilige wissenschaftliche Publikation rezipiert und sich anschließend schriftlich darüber äußert. Der Rezensent komprimiert, referiert und bewertet dabei das wissenschaftliche Handeln des Verfassers und die wissenschaftssprachliche Darstellung dieses Handelns in der Publikation. Er will den Leser zur Übernahme bestimmter Einschätzungen bewegen, und zwar »mithilfe von wissensbezogenen, rationalen und erfahrungsgeleiteten Argumenten «, so Fandrych/Thurmair (2011: 30), die die Rezension als ein Beispiel für die Gruppe der wissensbezogenen Texte aufführen. Der (wissenschaftliche) Leser leistet die Auseinandersetzung mit dem Primärtext zunächst vermittelt über die Rezension. Sie dient ihm zur schnellen Orientierung, als erster Zugang zu dem wissenschaftlichen Text und als Entscheidungshilfe für oder gegen eine ausführliche Lektüre desselben. Die Rezension beeinflusst folglich sowohl die Intensität der Auseinandersetzung mit dem Primärtext als auch seine Einordnung in den allgemeinen und den individuellen Forschungszusammenhang des Lesers. ${ }^{1}$

Die Textart ist seit den späten 1970er Jahren wiederholt zum Gegenstand mehrheitlich empirischer wissenschaftlicher Arbeiten geworden. Dabei stehen u. a. die folgenden Phänomene im Mittelpunkt des wissenschaftlichen Interesses:

- der Textbauplan und die Bestimmung der konstitutiven Teiltexte (Dallmann 1979, Foschi Albert 2009, Hutz 2001, Liang 1991, Ripfel 1997);

${ }^{1}$ Die wissenschaftliche Rezension ist allerdings nicht nur Teil der wissenschafts- und fachinternen Kommunikation, sondern kann auch an andere Rezipienten gerichtet sein, etwa wissenschaftlich ausgebildete Praktiker und Studierende. Ein Beispiel dafür sind die Rezensionen des jährlich erscheinenden Bandes »Für Sie gelesen« der Zeitschrift Info DaF. 
- die Art der rezensierten Inhalte (Foschi Albert 2009, Ripfel 1997, Zillig 1982);

- die vom Rezensenten an die Rezensionsvorlage angelegten Bewertungskriterien bzw. Wertmaßstäbe (Foschi Albert 2009, Zillig 1982);

- Handlungsmuster (Pätzold 1986);

- sprachliche Mittel des Referierens, des Bewertens, der Kritikverstärkung und -relativierung (Dallmann 1979, Dalmas 2001a/b, Foschi Albert 2009, Hutz 2001);

- Aspekte der Interpersonalität bzw. die Perspektivierung des Rezensenten im Text (Hutz 2001, Piitulainen 2001);

- die Rolle von Begründungen beim Bewerten (Wiegand 1983).

Zahlreiche Analysen sind sprachvergleichend angelegt: deutsch-englisch/amerikanisch (Hutz 2001), deutsch-chinesisch (Liang 1991), deutsch-französisch (Dalmas 2001b), deutsch-italienisch (Foschi Albert 2009), deutsch-finnisch (Piitulainen 2001) und deutsch-albanisch (Kristo 2010).

Viele der genannten Forschungsarbeiten versuchen darüber hinaus, eine Bestimmung der Funktionen und Sprachhandlungen der Textart zu leisten. Es herrscht dabei weitgehend Konsens darüber, dass in Rezensionen Inhalte dargestellt und beurteilt werden. Häufig ist von den Textfunktionen Informieren und Bewerten die Rede (Dallmann 1979, Foschi Albert 2009, Hutz 2001, Liang 1991, Zillig 1982, der allerdings von Textakten spricht), teilweise auch vom Beschreiben und Bewerten (Ripfel 1997). ${ }^{1}$

Aus der wissenschaftssprachdidaktischen Perspektive, die in diesem Artikel eingenommen wird, erscheint die funktionale Trennung von Informieren und Bewerten als ungünstig, suggeriert sie Studierenden und angehenden Wissenschaftlern doch, dass man in Rezensionen Informationen und Wertungen trennen könne bzw. sollte. Tatsächlich lässt sich das Informieren allerdings nur selten als »objektives« Informieren von Bewertungshandlungen abgrenzen, worauf auch Dallmann (1979: 63), Hintze (1989: 138) und Hutz (1991: 112) hinweisen. Schlüssiger scheint es deshalb, eine Reihe von primär gegenstandsbezogenen sprachlichen und mentalen Handlungen des Rezensenten beim Verfassen einer Rezension anzunehmen, z. B. das Erkennen, Auswählen und Gewichten von zentralen

${ }^{1}$ Bei genauerem Hinsehen zeigen sich insbesondere in Bezug auf die Darstellung der Inhalte in Rezensionen einige terminologische und kategoriale Unterschiede. Hutz (2001: 112) geht beispielsweise davon aus, dass die »Grundfunktionen « Informieren und Bewerten durch sogenannte Kommunikationsverfahren realisiert werden - das Informieren etwa, indem berichtet, beschrieben, referiert, zitiert und zusammengefasst wird. Ripfel (1997) dagegen betrachtet das Beschreiben als eigenständige Textfunktion. Pätzold (1986: 90) wiederum vernachlässigt die inhaltliche Darstellung durch den Rezensenten fast völlig und sieht die Funktion der Textart in der »Entwicklung bestimmter Wertvorstellungen« beim Leser. 
Wissenselementen, das Verarbeiten von argumentativen Zusammenhängen, das Zusammenfassen und Bewerten dieser Wissenselemente und Zusammenhänge, das Referieren in adäquater sprachlicher Form usw. Das Informieren lässt sich dann als übergreifende Textfunktion bestimmen, die auch explizite Kritik als Ergebnis von Bewertungen umfasst. Dieses Informieren ist ein rezipientenbezogenes Informieren, das dem Leser die Selektion relevanten Wissens und die Einordnung einer Publikation in den Forschungszusammenhang ermöglicht.

Die mentalen und sprachlichen Handlungsanforderungen beim Verfassen einer Rezension sind u. E. also deutlich komplexer, als es die funktionale Unterscheidung in Informieren/Beschreiben und Bewerten aus der Forschungsliteratur nahelegt. Nichtsdestotrotz lassen sich in Rezensionen i.d.R. zwei Teile ausmachen, in denen bestimmte Handlungen dominieren. Bei der inhaltlichen Darstellung ist das eine sprachlich-mentale Tätigkeit, die als ZUSAMMENFASSENDES REFERIEREN bezeichnet werden soll. Anders als Informieren oder Beschreiben benennt das zusammenfassende Referieren deutlicher das spezifische mentale und sprachliche Handeln von Rezensenten, nämlich die komprimierende Wiedergabe von Inhalten unter Bezugnahme auf den Verfasser und sein wissenschaftssprachliches Handeln. Unter BEWERTEN verstehen wir eine komplexe sprachliche Handlung, bei der das Gelesene zu bestimmten Kriterien ins Verhältnis gesetzt und an diesen gemessen wird. Diese Kriterien finden direkt oder indirekt in der sprachlichen Realisierung des Bewertungshandelns ihren Niederschlag. Das Bewerten ist fester Bestandteil des Schlussteils der Rezension, aber eben nicht auf diesen Teiltext beschränkt, sondern in der gesamten Rezension z. B. durch die Auswahl und Hervorhebung bestimmter Inhalte präsent.

Im Zusammenhang mit universitären Lehr-Lern-Situationen und der Vermittlung des Deutschen als (fremder) Wissenschaftssprache ist die Textart Rezension bisher kaum untersucht worden (anders als üblichere studentische Text- und Diskursarten wie die Seminararbeit, die Zusammenfassung, das Referat usw.). ${ }^{1}$ Dieser Mangel an Forschung zum Potential der Rezension im Prozess der Wissenschaftssozialisation und in der Wissenschaftssprachvermittlung überrascht insofern nicht, als wissenschaftliche Rezensionen Expertentexte sind. Sie werden in der Regel von Wissenschaftlern, die eine profunde Kenntnis des Gegenstands haben, und nicht von Studierenden geschrieben; sie werden zudem nur selten als studentische Leistung im Studium verlangt. Warum sollten Studierende also eine Textart einüben, die sie allenfalls selten produzieren müssen? Betrachtet man

1 Feilke/Lehnen (2011) stellen allerdings ein universitäres Schreibprojekt vor, bei dem Studierende einen wissenschaftlichen Artikel rezensieren und dabei lernen, Inhalte wissenschaftlich zu referieren, eine Bewertung argumentativ vorzubereiten usw. Pätzold (1986) plädiert für das Lehren textartspezifischer Handlungsmuster (z. B. zur Rezension) im studienbezogenen Deutschunterricht. 
allerdings die Handlungsspezifik der Textart, lässt sich ihre Eignung für das studentische Schreiben durchaus erkennen: Sie bringt den studentischen Autor dazu, wissenschaftliches Wissen kritisch zu verarbeiten und komprimiert darzustellen (zusammenfassendes Referieren) und verlangt eine Positionierung zum wissenschaftlichen Wissen (Bewerten). Es sind diese textkonstitutiven sprachlichen Handlungen, die die Textart für das studentische Schreiben interessant machen. Sie fördern das Verständnis von wissenschaftlichem Wissen als strittigem Wissen und damit die Ausbildung einer eristischen Schreibkompetenz.

Das studentische sprachliche Handeln beim Verfassen einer Rezension soll im Folgenden ausführlicher und mit Bezug auf einige Ergebnisse der Schreib- und Wissenschaftssprachforschung ${ }^{1}$ dargestellt werden:

(a) Wer rezensiert, muss zunächst den zu rezensierenden Text rezipieren. Dabei sind von den Studierenden komplexe mentale Verarbeitungsleistungen zu erbringen, etwa das Verstehen argumentativer Zusammenhänge und das Erkennen der wissenschaftlichen Position des Autors. Die Rezeption ist dabei immer schon ein Teil des gesamten Bewertungsprozesses. Redder (2002) spricht im Hinblick auf diesen Verarbeitungs- und Bewertungsprozess vom fachspezifischen wissenschaftlichen Einschätzen, das Studierende nur langsam und in der Regel erst gegen Ende ihrer Wissenschaftssozialisation erwerben. Erst in der letzten der fünf »Etappen der Wissenskonstellationen und der Aneignung des Einschätzens" (19, Hervorhebung i. O.), die Redder annimmt, sind Studierende dazu in der Lage, sich selbständig wissenschaftliches Wissen anzueignen und es so weiterzuverarbeiten, dass es für ihr eigenes wissenschaftliches Handeln nutzbar wird. Das Verfassen von Rezensionen kann ihnen bei diesem Aneignungsprozess des wissenschaftlichen Einschätzens helfen, denn es fördert die Fähigkeit, sich einen Forschungsbereich zu erarbeiten sowie einzelne Forschungsarbeiten zueinander in Bezug zu setzen und in den Forschungszusammenhang einzuordnen. ${ }^{2}$

1 Von großer Bedeutung in Bezug auf den wissenschaftlichen Schreiberwerb ist die Arbeit von Steinhoff (2007), der an einem großen Korpus von 296 studentischen Seminararbeiten aus allen Studienphasen des traditionellen Magisterstudiums zeigen kann, wie sich wissenschaftliche Textkompetenz im Laufe der Wissenschaftssozialisation entwickelt. Seinem Modell zufolge durchlaufen Studierende einen Annäherungsprozess an wissenschaftliches Schreiben, der die Erwerbsphasen der Transposition, Imitation und Transformation umfasst, bevor ggf. eine kontextuelle Passung, d.h. ein domänentypisches und kontextadäquates Schreiben, erreicht wird. Diese Phasen und ihre sprachlichen Merkmale (Imitation, Formulierungsbrüche) sind auch im Hinblick auf die Analyse von studentischen Rezensionen von Bedeutung - dort finden sich ähnliche Phänomene wie in den von Steinhoff analysierten Seminararbeiten.

2 Für eine grundlegende Bestimmung der Teilschritte des Einschätzens siehe auch Rehbein (1977: 143). 
(b) Wer rezensiert, muss das Gelesene zusammenfassend referieren und bewerten können. Das gelesene und mental verarbeitete Wissen muss in den eigenen Text integriert und explizit beurteilt, es muss weiterverarbeitet werden. Diesen Bereich, in dem Wissen ausgewählt, verdichtet, umformuliert, umgestellt oder auch ausgelassen wird, untersucht Stezano Cotelo (2008) für studentische Seminararbeiten, insbesondere von ausländischen Studierenden. Sie identifiziert drei charakteristische Verfahren, die Studierende bei der Wissensverarbeitung anwenden und die in dieser Reihenfolge auch grob den Stand der Wissenschaftssozialisation widerspiegeln. Beim ersten Verarbeitungstyp werden hauptsächlich Textteile ausgewählt und zitiert (»Null-Reformulierung «, 260). Eine deutlich größere mentale Verarbeitungsleistung erfordert der zweite Typ, eine Kombination aus zitierendem sprachlichen Handeln und Umformulieren. Für den dritten Verarbeitungstyp - simultanes Umformulieren und Zusammenfassen - findet Stezano Cotelo nur wenige Belege, was auf die Komplexität des zusammenfassenden und umformulierenden Handelns hindeutet.

Die wissenschaftliche Rezension verlangt ihrem Verfasser nun in der Regel ein geringeres Maß an synthetisierendem wissenschaftlichen Schreiben als die Seminararbeit ab. Der Verfasser bezieht sich hauptsächlich auf eine Publikation und kann sich an der Textstruktur dieser Publikation orientieren. Trotzdem muss er kompetent umformulieren und zusammenfassend referieren können, denn anders lassen sich eine Monographie oder ein Sammelband nicht auf wenigen Seiten leserfreundlich darstellen. Im Modul Deutsch als Wissenschaftssprache lassen wir die Masterstudierenden Schreibaufgaben zur Vorentlastung bearbeiten (kurze Zusammenfassungen, Exzerpte, kommentierte Bibliographie, vgl. Kapitel 1), damit sie dieser Anforderung gewachsen sind. Trotzdem wird sich an den Korpusbelegen in Abschnitt 3 zeigen, dass die mentalen Verarbeitungsleistungen beim Schreiben einer Rezension auch für fortgeschrittene Studierende eine sehr große Herausforderung darstellen.

Die wissenschaftliche Bewertung des im Primärtext präsentierten Wissens erfordert nicht nur 1) die Aufnahme von Textinformationen bzw. die Rekonstruktion der argumentativen Zusammenhänge des Primärtextes, sondern 2) u. a. auch ein umfangreicheres (inhaltliches und methodisches) Wissen über den Forschungsbereich, in den sich die Publikation einordnet - zu diesem muss das im Primärtext präsentierte Wissen ins Verhältnis gesetzt werden. Darüber hinaus setzt es 3) beim wissenschaftlichen Autor ein Verständnis von in der Wissenschaftsgemeinschaft akzeptierten und für den Forschungszusammenhang relevanten Bewertungskriterien voraus, an denen der Primärtext zu messen ist (vgl. hierzu Steinhoff 2007: 361ff.). Wissenschaftliche Werte wie Richtigkeit, Vollständigkeit und Differenziertheit eignen sich Studierende, so Steinhoff, erst in einem längeren Prozess an. In Bezug auf die Nutzung der Rezension als studentische Textart stellt sich hier demzufolge auch die Frage, welche (wissenschaftlichen) Kriterien studentische 
Autoren an Rezensionsvorlagen anlegen und in welchem Umfang sie damit den Konventionen der Textart entsprechen (wie sie etwa Zillig 1982 beschreibt, vgl. ausführlicher Abschnitt 3.2. zum Bewerten in Rezensionen).

Systematische Unterschiede zwischen deutsch- und fremdsprachigen Studierenden scheinen in Bezug auf die Wissensverarbeitung nicht $\mathrm{zu}$ bestehen. Stezano Cotelo (2008: 273) kommt zu dem Ergebnis, dass ausländische Studierende zwar größere Schwierigkeiten mit der Textoberfläche/den sprachlichen Mitteln hätten und zu Beginn des Studiums deutlich stärker zum Zitieren neigen würden, dass aber »die Hauptschwierigkeiten, die im Umgang mit der wissenschaftlichen Literatur liegen, kaum durch die Fremdsprache beeinflusst « würden. Dieser Befund schließt allerdings nicht aus, dass Studierende von wissenschaftlichen Denk- und Schreibtraditionen geprägt sind, die sich unterschiedlich stark auf das sprachliche Handeln, die Verarbeitung von wissenschaftlichem Wissen und insbesonders auch auf das Bewerten/Kritisieren von Wissenschaft und Wissenschaftlern auswirken (vgl. Ehlich 1995, 2003). Bei der Einführung nichtdeutscher Studierender in das Schreiben einer wissenschaftlichen Rezension sollten spezifische Diskurstraditionen/-konventionen in jedem Fall im Blick behalten werden. Die Mehrzahl der oben aufgeführten kontrastiven Analysen arbeitet solche Diskurskonventionen zwischen deutschen und anderssprachigen Rezensionen heraus und schärft den Blick für das Spezifische der Textart in unterschiedlichen Wissenschaftstraditionen. In mehreren Untersuchungen wurde beispielsweise eine Tendenz zu expliziter, inhaltsbezogener Kritik in deutschen Rezensionen festgestellt - nichtdeutsche Studierende müssen also ggf. zum deutlichen Bewerten ermuntert werden und dieses explizite Bewerten üben. ${ }^{1}$

(c) Wer rezensiert, muss schließlich auch die sprachliche Oberfläche seines Textes rezensionsspezifisch gestalten können, indem er $u$.a. die alltägliche Wissenschaftssprache adäquat verwendet. Für das zusammenfassende Referieren benötigt der Autor z. B. sprachliche Mittel des Diskurses über Forschung (Sprechhand-

${ }^{1}$ Hutz (2001) kann etwa belegen, dass deutsche Rezensenten häufiger kritikverstärkende Ausdrücke verwenden, was er als Ausdruck einer größeren Sachorientierung deutet. Foschi Albert (2009) findet heraus, dass deutsche und italienische Rezensenten z. T. anderen Bewertungskriterien folgen, dass italienische Rezensenten generell vorsichtiger bewerten und sich dabei - wenn überhaupt - auf formale Mängel konzentrieren. Wir können die Frage nach dem Einfluss bestimmter wissenschaftlicher Diskurstraditionen auf das wissenschaftliche Schreiben an dieser Stelle und für die heterogenen Studierendengruppen im Leipziger Wissenschaftssprachmodul nicht ausführlich erörtern, verweisen aber auf die einschlägigen Arbeiten z. B. von Kaiser (2002), verschiedene Beiträge in Ehlich/Steets (2003) sowie auf die bereits genannten kontrastiven Analysen zur Rezension. 
lungsverben wie fragen, argumentieren, behaupten) und Konjunktionen/Konjunktionaladverbien (zwarlaber, allerdings) zur Wiedergabe argumentativer Zusammenhänge. Zum Repertoire an wertenden sprachlichen Mitteln gehören wertende Adjektive (irrelevant, gering), Nomen (Verdienst, Schwäche) und Verben (nachweisen, versäumen, verkennen), Adverbien (leider, besonders, überaus, kaum, mitunter), Abtönungspartikeln (wohl), Modalverben, konzessive Satzstrukturen, Konjunktivformen u.a.

\section{Empirische Analyse: Zentrale wissenschaftssprachliche Handlungen in Rezensionen}

Aus dem Modul »Deutsch als Wissenschaftssprache« wurden 20 studentische Rezensionen ${ }^{1}$ im Hinblick auf die wissenschaftssprachlichen Handlungen zusammenfassendes Referieren und Bewerten analysiert und an geeigneten Stellen mit zwei Expertenrezensionen verglichen. Den Ausgangspunkt für die Analyse beider wissenschaftssprachlicher Sprechhandlungen bilden die im vorangegangenen Kapitel dargestellten Anforderungen, denen ausländische Studierende beim Verfassen einer Rezension gerecht werden müssen.

Im ersten Teil der empirischen Untersuchung werden zwei studentische Texte im Hinblick auf die Auswahl und Gewichtung von Inhalten beim zusammenfassenden Referieren, die Verarbeitung von Wissen aus der Rezensionsvorlage und die Einhaltung der Rezensentenperspektive untersucht. Die Beschränkung auf zwei Fallanalysen liegt darin begründet, dass beiden Texten im Gegensatz zum restlichen Lernerkorpus die gleiche Rezensionsvorlage zugrunde liegt. Eine Expertenrezension wird als Vergleichstext herangezogen, um unterschiedliche Herangehensweisen dreier Rezensenten beim zusammenfassenden Referieren zu illustrieren. Durch die Gegenüberstellung soll außerdem verdeutlicht werden, dass qualitative Unterschiede beim zusammenfassenden Referieren nicht allein entwicklungsbedingt sind, sondern auch in Abhängigkeit von individuellen Schreibstilen und inhaltlichen Schwerpunktsetzungen gesehen werden müssen. In Anbetracht dessen sind Expertenrezensionen, wie es der Vergleich mit studentischen Texten beispielhaft veranschaulichen soll, nicht zwangsläufig als Modell für die Vermittlung der Textart und ihrer konstitutiven wissenschaftssprachlichen Handlungen geeignet. Aufgrund der beschränkten Zahl der Texte handelt es sich bei dem Vergleich um eine rein qualitative Analyse mit dem Ziel, die untersuchten Phänomene detailliert zu beschreiben.

1 Es handelt sich um Rezensionen von Studierenden aus Ägypten, Brasilien, Mexiko, Kolumbien, Spanien und Belgien. 
Im zweiten Teil der Korpusanalyse steht die Frage im Vordergrund, ob und wie die Studierenden dazu in der Lage sind, die Rezensionsvorlage zu bewerten. Neben dem Umfang, der sprachlichen Realisierung und der Angemessenheit von Bewertungen sind auch die der Sprachhandlung zugrunde liegenden Bewertungskriterien sowie die Begründungen der Bewertungen Gegenstand der Untersuchung. Hier werden alle 20 studentischen Rezensionen einbezogen und neben Fallanalysen auch quantitative Aspekte bei der Auswertung berücksichtigt. Zudem wird eine zweite Expertenrezension in Auszügen als Vergleichstext analysiert, um die wissenschaftssprachlichen Herausforderungen, mit denen ausländische Studierende beim Bewerten von Rezensionsvorlagen konfrontiert sind, zu illustrieren.

Sowohl bei der Untersuchung des zusammenfassenden Referierens als auch des Bewertens wird ein datengeleitetes Verfahren verwendet, bei dem nach den Leitsätzen der Korpusanalyse induktiv aus den untersuchten Texten heraus Erkenntnisse über den Untersuchungsgegenstand gewonnen werden. Vorannahmen werden bei diesem Verfahren - so weit es möglich ist - ausgeblendet und die aus der Analyse gewonnenen Ergebnisse werden auf das untersuchte Korpus beschränkt (vgl. Glück 2005: 357 zur Korpusanalyse).

\subsection{Zusammenfassendes Referieren}

Um das zusammenfassende Referieren als Sprechhandlung vergleichend untersuchen zu können, war es nötig, sich auf die studentischen Rezensionen zu beschränken, denen die gleiche Rezensionsvorlage zugrunde lag. Da Rezensionsvorlagen von Studierenden im Modul »Deutsch als Wissenschaftssprache« immer im Einklang mit ihrem Forschungsinteresse (Abschlussarbeit oder Modularbeit) gewählt werden, ist die Bandbreite der begutachteten Werke entsprechend groß. In unserem Korpus gibt es daher nur zwei studentische Rezensionen, die den gleichen Text als Grundlage haben: Rösler, Dietmar (2007): E-Learning: Eine kritische Einführung. (2. Aufl.) Tübingen: Stauffenburg. Als Vergleichstext wurde eine Expertenrezension von Florin (2008) hinzugezogen. Aus Platzgründen beschränkt sich der Vergleich auf das sechste Kapitel (»Feedback und Tutoring«), das zunächst kurz zusammengefasst werden soll.

Rösler beschäftigt sich in diesem Kapitel mit verschiedenen Formen der OnlineRückmeldung. Er geht zunächst auf Formen des programmierten Feedbacks ein. Er nennt Vor- und Nachteile des digitalen programmierten Feedbacks, verdeutlicht diese mit positiven und problematischen Softwarebeispielen und erläutert Ansätze für intelligentes computergestütztes Feedback/Sprachenlernen (ICALL, pädagogische Agenten). Im zweiten Teil des Kapitels widmet er sich den Aufgaben des Tutors beim computergestützten Sprachenlernen, stellt vier verschiedene Tutoring-Konzepte und eine Instant-Messaging-Software als neues 
Beratungswerkzeug vor und diskutiert abschließend die Rolle des Lehrers in diesen neuen Lehr- und Lernformen. Röslers Position zum Online-Feedback ist positiv-kritisch: Er spricht ihm zwar eine zentrale Bedeutung für die langfristige Akzeptanz von Lernsoftware zu, fordert aber verbesserte Feedback-Lösungen für geschlossene Aufgaben/Übungen und äußert deutliche Zweifel an einer schnellen Verbesserung intelligenter Feedbackmöglichkeiten für halboffene und offene Aufgaben.

Die drei folgenden Textabschnitte sind Zusammenfassungen des sechsten Kapitels in den besagten Rezensionen:

B1: Stehen im traditionellen Unterricht die Lehrenden unmittelbar zur Verfügung, so erfordert das Arbeiten mit digitalen Medien neue Formen der Rückmeldung, Betreuung und der Lernberatung. Wenn man an die Feedbacksysteme mancher Lernprogramme denkt, weiß man, dass dieses Problem nur unbefriedigend gelöst ist. Entweder erfordert ein sinnvolles Feedback enormen Programmieraufwand oder ein sinnvoll organisiertes Tutorensystem, wobei in beiden Fällen neben Fragen des Aufwandes und der Machbarkeit auch profane Finanzierungsfragen ins Spiel kommen. (Auszug Florin 2008: 328)

B2: Im sechsten Teil wird der Frage nach der Tutor-Rolle in Bezug auf Feedback und seine Relation zum Lehrer eingegangen. Dass es verschiedene Variante von Tutoren in digitalen Medien wie z. B. impliziter Tutor Onlinetutor und Tutor als Lernberater wird schon auch hingewiesen. Bezogend auf die Bedeutung des Feedback für das Fremdsprachenlernen mit digitalen Medien erklärt Rösler: »Gerade die Tendenz, digitales Lernmaterial sehr weitgehend mit dem Selbstlernen in Verbindung zu bringen, macht das Feedback für das Fremdsprachenlernen mit digitalen Medien so bedeutsam " (Rösler 2004, 178). Sowohl die Vor- und Nachteile des digitalen Feedback als auch das programmierte Feedback werden nicht außer Acht gelassen. Allerdings wird die Rolle des Tutors in verschiedenen Kontexten wie z. B. Chat und Tandem erörtert. (Auszug Student A.)

B3: Im sechsten Kapitel zeigt Rösler die immer noch bestehende Forschungslücke im Bereich der digitalen Medien auf, die bis heute immer noch nicht geschlossen wurde. Es ist das Problem des elektronischen Feedbacks, wobei er die Bedeutung des digitalen Feedbacks für das Selbststudium hervorhebt. Auch stützt sich der Autor hier wieder auf unterschiedliche Literatur und Untersuchungen, die sich mit diesem Forschungsdesiderats beschäftigen. Er listet wieder die Möglichkeiten des elektronischen Feedbacks auf und zeigt dabei auch die Mängel an, die sich besonders im Bereich des freien Schreibens im Netz bestehen und stellt also Lösung dafür die intelligenten Analysesysteme vor, mit denen sich die Forscher in den nächsten Jahren noch beschäftigen sollen. Auch sieht der Autor in den pädagogischen Agenten eine Teillösung des Problems. Noch bleibt ein wichtiger Teil des natürlichen Feedbacks im Bereich der digitalen Medien unberührt und zwar das nonverbale Feedback durch Mimik und Gestik, dass in diesem Bereich schwer zu erreichen ist. Außerdem gibt Rösler den online Tutoren einige Hinweise zum Umgang mit dem elektronischen Feedback. (Auszug Studentin S.)

Die drei Rezensionsauszüge differieren auf verschiedenen Ebenen. Ein erster offensichtlicher Unterschied besteht im Umfang der Darstellung von Röslers Kapitel »Feedback und Tutoring «. Während Florin nur knapp und indirekt darauf eingeht - er betont an anderer Stelle, dass für ihn »die beiden ersten [Kapitel] die 
wichtigsten« (326) seien -, widmen die beiden Studierenden dem Kapitel mehr Aufmerksamkeit. Neben quantitativen Aspekten unterscheiden sich die drei Rezensionsausschnitte jedoch auch hinsichtlich der Auswahl und Gewichtung von Inhalten (3.1.1), der Verarbeitung von Wissen (3.1.2) und der Verfasserreferenz (3.1.3).

\subsubsection{Auswahl und Gewichtung von Inhalten}

Der propositionale Gehalt von wissenschaftlichen Rezensionen ist nach Ripfel (1997: 491) nicht nur in Abhängigkeit vom rezensierten Werk zu sehen, sondern auch in Abhängigkeit davon, »was der Rezensent mit Blick auf seinen Adressatenkreis für beschreibungs- und bewertungswürdig hält«. Wie unterschiedlich die drei Rezensenten Sachverhalte aus der Rezensionsvorlage auswählen und sie gewichten, wird in den obigen Auszügen deutlich.

Florin hebt in seiner Zusammenfassung die von Rösler in Kapitel 6 geäußerte Kritik an programmiertem Feedback und die generell mit digitalen Rückmeldungen einhergehenden Herausforderungen hervor. Seine Ausführungen sind in erster Linie praxisorientiert, indem er etwa den Arbeitsaufwand, die Realisierbarkeit und finanzielle Aspekte des elektronischen Feedbacks betont. Die von Rösler skizzierten Feedbackarten und die Möglichkeiten, die sich durch digitales Feedback ergeben, finden in seiner Zusammenfassung ebenso wenig Berücksichtigung wie dessen Desiderata und Ausblicke.

Im Gegensatz zu Florin sieht Student A. in seiner Darstellung die unterschiedlichen Rollen des Online-Tutors in digitalen Lernumgebungen als zentralen Gegenstand des Kapitels an. Ein entsprechender Hinweis findet sich in seiner nicht-linearen Abhandlung sowohl am Anfang als auch am Ende des Abschnitts wieder. Auf den ersten Teil des Kapitels, die Vor- und Nachteile des programmierten Feedbacks, geht er hingegen nur am Rande ein, indem er erwähnt, dass diese auch nicht außer Acht gelassen werden. Die von Rösler vorgenommene Zweiteilung des Kapitels in programmiertes Feedback und Online-Tutoren und die Gewichtung dieser Inhalte werden dadurch nicht deutlich. In den Vordergrund rückt Student A. zudem die Bedeutung des digitalen Feedbacks im Kontext des autonomen Lernens, indem er ein entsprechendes direktes Zitat Röslers einbindet und ihm damit eine besondere inhaltliche Bedeutung zuspricht.

Den roten Faden der Inhaltszusammenfassung von Studentin S. bilden die mit dem elektronischen Feedback verbundenen Schwierigkeiten und potentielle Lösungsansätze, die Rösler in seinem Kapitel anspricht. Ihre Ausführungen, die im Vergleich zu den anderen beiden am ausführlichsten sind, beziehen sich zuvorderst auf den ersten Teil des sechsten Kapitels. Röslers Überlegungen zu Online-Tutoren werden hingegen nur im letzten Satz und zudem wieder unter dem spezifischen Fokus des digitalen Feedbacks erwähnt. Die hier vorgenom- 
mene einseitige Gewichtung ist somit diametral entgegengesetzt $\mathrm{zu}$ der von Student A. Gemeinsam ist beiden Inhaltsangaben jedoch, dass auch Studentin S. den Zusammenhang zwischen elektronischem Feedback und autonomem Lernen aufgreift.

Zusammenfassend lässt sich festhalten, dass alle drei Rezensenten zum einen Teilaspekte des Kapitels in den Vordergrund ihrer Inhaltsangabe stellen und zum anderen konstitutive Elemente des Kapitels unberücksichtigt lassen. Auffällig ist in diesem Zusammenhang auch, dass in keinem der drei Rezensionsauszüge die Makrostruktur des Kapitels, die sich durch eine Zweiteilung in programmiertes Feedback und Online-Tutoring auszeichnet, aufgegriffen wird.

Ein wesentlicher Unterschied zwischen der Expertenrezension und den beiden studentischen Texten besteht im Grad der Abstraktion bei der Komprimierung des gelesenen Inhalts. Während Florin sich mit seinen Äußerungen weit von der Textvorlage entfernt, zeichnen sich die beiden studentischen Texte durch Detailinformationen über das Kapitel aus, wobei die Motivation für die inhaltliche Auswahl nicht immer nachvollziehbar ist. Insgesamt lassen sich in den Rezensionen zwei unterschiedliche Blickwinkel erkennen: Während die studentischen Rezensionen eher eine wissenschaftliche Perspektive einnehmen, ist Florins Rezension stärker praxisorientiert. Aus der Perspektive des Lesers, der sich über Röslers Publikation informieren möchte, ist die Darstellung des Kapitels bei Florin u.E. die unergiebigste, ähnelt sie doch eher einem eigenständigen Beitrag zum Thema als einer bewertenden Wiedergabe der Rezensionsvorlage.

\subsubsection{Verarbeitung von Wissen}

Im Folgenden sollen zwei Bereiche der Wissensverarbeitung exemplarisch näher betrachtet werden: die Einbindung von Zitaten und syntaktische Mittel der Textkohäsion/-kohärenz. ${ }^{1}$

Probleme mit der Wiedergabe fremden Wissens zeigen sich in Texten häufig daran, in welchem Umfang und auf welche Weise direkte Zitate in den Text eingebunden werden. Steinhoff (2007) ordnet das direkte Zitieren in seinem

1 Wie Rezensenten im Zuge des Lese- und Schreibprozesses das in der Rezensionsvorlage angelegte Wissen verarbeiten, lässt sich nicht direkt beobachten. Vielmehr müssen solche kognitiven Vorgänge mithilfe verschiedener Verfahren rekonstruiert werden. In der vorliegenden Arbeit werden in Anlehnung an das Vorgehen von Steinhoff (2007) und Stezano Cotelo (2008) Rückschlüsse über Verarbeitungsschwierigkeiten allein auf der Grundlage von Textanalysen gezogen. Da keinerlei Schreibprozessdaten vorlagen, ist die Reichweite der Untersuchungsergebnisse mit den entsprechenden Einschränkungen verbunden, die eine reine Textanalyse mit sich bringt (vgl. auch Heine/Schramm 2007: 167 zur Schwierigkeit, aus Schreibprodukten Aussagen über deren Entstehungsprozesse abzuleiten). 
Modell der Entwicklung von wissenschaftlicher Textkompetenz der Imitationsphase zu. Damit verortet er es auf der untersten Stufe auf dem Weg zur wissenschaftlichen Textkompetenz. Die Fähigkeit, den propositionalen und illokutiven Gehalt anderer Positionen mit eigenen Worten angemessen wiederzugeben, wird nach Steinhoff (2007) von Studierenden erst spät im Laufe der Wissenschaftssozialisation erworben. Wie in Abschnitt 2 bereits erwähnt, sieht auch Stezano Cotelo (2008) das direkt zitierende sprachliche Handeln als Indikator für eine studentische Unerfahrenheit bei der Wissensverarbeitung.

Die drei hier betrachteten Rezensionen unterscheiden sich im Hinblick auf die Einbindung direkter Zitate deutlich voneinander. Während Florin in seine gesamte Rezension zwei direkte Zitate integriert, macht Studentin S. gar keinen Gebrauch von direkten Zitaten. Student A. fügt in seine Rezension wiederum insgesamt fünf direkte Zitate ein, deren Einbindung in den Text jedoch meist nur bedingt nachvollziehbar ist, weil sie beispielsweise redundant sind oder gegen Regeln der Textkohärenz und/oder -kohäsion verstoßen wird. In den hier betrachteten Auszug fügt Student A. ein direktes Zitat Röslers ein, während Studentin S. den gleichen Inhalt paraphrasiert und Florin gar nicht auf den entsprechenden Punkt eingeht.

B4: Bezogend auf die Bedeutung des Feedback für das Fremdsprachenlernen mit digitalen Medien erklärt Rösler: »Gerade die Tendenz, digitales Lernmaterial sehr weitgehend mit dem Selbstlernen in Verbindung zu bringen, macht das Feedback für das Fremdsprachenlernen mit digitalen Medien so bedeutsam" (Rösler 2004, 178). (Student A.)

B5: Im sechsten Kapitel zeigt Rösler die immer noch bestehende Forschungslücke im Bereich der digitalen Medien auf, die bis heute immer noch nicht geschlossen wurde. Es ist das Problem des elektronischen Feedbacks, wobei er die Bedeutung des digitalen Feedbacks für das Selbststudium hervorhebt. (Studentin S.)

An beiden Zitierweisen lassen sich Probleme bei der Wissensverarbeitung erkennen. So bezieht sich das von Student A. angeführte Zitat im Original nicht allein, wie im Einleitungssatz suggeriert wird, auf die Relevanz des Feedbacks für das Sprachenlernen in digitalen Lernumgebungen. Vielmehr hebt Rösler im Original mithilfe der Fokuspartikel gerade auch den Zusammenhang zwischen autonomem Lernen und elektronischen Lernmaterialien hervor und sieht diesen als einen Grund für die wichtige Rolle des elektronischen Feedbacks. Der Einleitungssatz deutet darauf hin, dass der propositionale Gehalt des Originaltextes nicht in seiner Gänze erfasst wurde. Studentin S. gelingt es an dieser Stelle besser, den Kern der Aussage mit eigenen Worten wiederzugeben. Sie paraphrasiert die im Original enthaltene Aussage sowohl auf der Äußerungsebene als auch im Hinblick auf den propositionalen Gehalt. Irritierend wirkt hingegen auf der Ebene der Textkohäsion ihre Einleitung des Teilsatzes durch das Konjunktionaladverb wobei, das hier möglicherweise in restriktiver Funktion verwendet werden soll. 
Hier wird versucht, durch den Konnektor zwei Gedanken miteinander zu verknüpfen, zwischen denen keine spezifische inhaltliche Beziehung besteht. Damit ist ein zweites Problem der Wissensverarbeitung angesprochen, nämlich Schwierigkeiten bei der Textverknüpfung. Diese treten insbesondere bei Student A. auf:

Sowohl die Vor- und Nachteile des digitalen Feedback als auch das programmierte Feedback werden nicht außer Acht gelassen. Allerdings wird die Rolle des Tutors in verschiedenen Kontexten wie z. B. Chat und Tandem erörtert.

Den abschließenden Satz leitet der Rezensent mit dem Konjunktionaladverb allerdings ein, obwohl es sich keineswegs um eine restriktive Aussage über Röslers Darstellung der Vor- und Nachteile des digitalen Feedbacks handelt, sondern vielmehr um eine weitere Information zur Rolle des Tutors. Der erste Satz offenbart ebenfalls Schwächen bei der Wahl der richtigen Konjunktion - sowohl ... als auch ...nicht statt weder ... noch - und zudem Verstehensschwierigkeiten bei der Rezeption Röslers, da digitales und programmiertes Feedback hier getrennt voneinander aufgeführt werden, obwohl Rösler (2007: 179) letzteres eher als eine Unterart der digitalen Rückmeldung darstellt.

In diesem kurzen Auszug offenbaren sich somit nicht nur Lücken in der Sprachbeherrschung, sondern die fehlerhaft verwendeten Mittel zur Textkohäsion lassen sich auch als Indikator für Probleme bei der Wissensverarbeitung werten.

\subsubsection{Verfasserreferenz/Rezensentenperspektive}

Anhand der drei Textauszüge soll nun verdeutlicht werden, wie unterschiedlich Rezensenten die illokutive Dimension des sprachlichen Handelns von Rösler wiedergeben. Folgende zwei Fragen stehen im Vordergrund der Analyse: Werden Handlungen des Verfassers im Original (korrekt) wiedergegeben und sprachlich markiert? Ist die Rezensentenperspektive durchgängig vorhanden, d.h. die Perspektive des zusammenfassenden Referierens und Bewertens?

Die sprachliche Markierung von Handlungen eines anderen Autors wird in Rezensionen meist durch den Einsatz von Sprechhandlungsverben realisiert. Unter Sprechhandlungsverben werden in Anlehnung an Fandrych (2002: 3) »alle solchen Verben verstanden, mit denen sprachliche Handlungen einfacher oder komplexer Natur benannt werden können «. Sie sind Teil der allgemeinen Wissenschaftssprache, die neben ihrer wissenschaftlichen auch über eine allgemeinsprachliche Bedeutung verfügen (beispielsweise erklären, hervorheben, sich auf etwas stützen).

Während in den beiden studentischen Arbeiten das wissenschaftliche Handeln Röslers mithilfe solcher Sprechhandlungsverben wiedergegeben wird, fehlen diese in Florins Rezensionsauszug. Dort ist der Bezug zu Röslers Kapitel nicht unmittelbar erkennbar, sondern er zeigt sich nur indirekt am Textaufbau der 
Rezension. Betrachtet man den Ausschnitt jedoch isoliert, dann ist unklar, ob es sich bei der Abhandlung zum elektronischen Feedback um Reflexionen des Rezensenten über das Thema oder die Wiedergabe von Röslers Positionen handelt. Neben dem Fehlen von Sprechhandlungsverben wird dieser Eindruck durch die Verwendung des unpersönlichen man verstärkt: Wenn man an die Feedbacksysteme mancher Lernprogramme denkt, weiß man, dass dieses Problem nur unbefriedigend gelöst ist. Die hörer- bzw. leserinklusive Passiversatzkonstruktion mit man lenkt vom Verfasser ab, auf den sich dieser Absatz eigentlich bezieht. Zudem sticht die Konstruktion Wenn man an [...] denkt, weiß man, [...] durch ihre Nähe zur mündlichen Sprache hervor. Insgesamt markiert Florin seine Position als Rezensent nicht als solche, da er in seinem Rezensionsausschnitt konsequent den Verfasser ausblendet und nicht dessen Handlungen beschreibt.

Dieses Phänomen zeigt sich auch in verschiedenen studentischen Rezensionen. In den beiden oben angeführten Ausschnitten ist dies allerdings nicht der Fall. Beide Studierenden nehmen deutlich eine Rezensentenperspektive ein und weisen ausdrücklich auf das Handeln des rezensierten Autors hin. Am explizitesten ist dabei Studentin S., die durchgängig mit Sprechhandlungsverben im Aktiv und dem Verweis auf den Autor (Rösler, er, der Autor) ihre Position als Rezensentin markiert. Student A. hingegen blendet durch die Verwendung des Vorgangspassivs den rezensierten Autor zwar meist aus, dennoch wird auch bei ihm durch den konsequenten Gebrauch von Sprechhandlungsverben und einen klaren Verweis auf den Autor innerhalb einer Aktivkonstruktion (erklärt Rösler) der Bezug zu Rösler deutlich.

Beide Auszüge zeichnen sich zudem dadurch aus, dass sie das sprachliche Handeln Röslers bis auf wenige Ausnahmen angemessen wiedergeben. Wenn Unsicherheiten auftreten, dann sind diese meist nicht im Zusammenhang mit dem Erkennen der Sprechhandlung zu sehen, sondern liegen auf anderen Ebenen, wie es Beispiel 3 von Studentin S. zeigt. So ist zwar das Sprechhandlungsverb stützen, mit dem Studentin S. darauf hinweist, dass Röslers Ausführungen auf anderen Quellen und Studien fußen, richtig gewählt. Es ist jedoch nicht notwendig, diese sprachliche Handlung des Autors zu erwähnen, da sie konstitutives Merkmal einer jeden wissenschaftlichen Arbeit ist. Eine weitere Besonderheit ihrer Inhaltsangabe ist, dass ihrer Meinung nach Rösler im Bereich der elektronischen Rückmeldungen eine Forschungslücke aufzeigt. Diese Sprachhandlung wird vom Autor nicht vollzogen, obgleich er an verschiedenen Stellen auf die Nachteile des Feedbacks eingeht. Unter Umständen ist diese fehlerhafte Wiedergabe durch die Studentin S. auf ein Missverständnis des Terminus Forschungslücke zurückzuführen, den sie in diesem Kontext mit »Problem « gleichsetzt.

Die Analyse der drei Rezensionsausschnitte verdeutlicht, wie unterschiedlich Inhalte aus einer Rezensionsvorlage verarbeitet und wiedergegeben werden können. Divergenzen bestehen auf allen drei untersuchten Ebenen: der Auswahl 
und Gewichtung von Inhalten, der Verarbeitung des gelesenen Textes und der Markierung der Rezensentenperspektive. Aufgrund der geringen Anzahl an untersuchten Rezensionen, der Beschränkung auf einen Rezensionsausschnitt sowie des spezifischen Lernhintergrunds der beiden studentischen Verfasser lassen sich zwar keine verallgemeinerbaren Aussagen über Schwierigkeiten ausländischer Studierender beim zusammenfassenden Referieren fremden Wissens treffen. Die Fallbeispiele werfen jedoch einige interessante Fragen auf, die sich im Rahmen der Vermittlung wissenschaftlicher Schreibkompetenz an ausländische Studierende thematisieren ließen: Wie müssen Inhalte einer Rezensionsvorlage ausgewählt und gewichtet werden, um den Leser der Rezension in komprimierter, aber ausreichender Form zu informieren? Wann und wie bietet sich die Einbindung direkter Zitate in Rezensionen an? Wie lässt sich die Argumentationsstruktur der Rezensionsvorlage beim zusammenfassenden Referieren erhalten? Wie stark muss ein Rezensent seine Verfasserrolle sprachlich markieren, um seine Ausführungen von der Wiedergabe fremden Wissens zu trennen?

\subsection{Bewerten}

Wie Steinhoff (2007) deutlich machen kann, finden sich textkritische Äußerungen in studentischen Hausarbeiten nur selten:
»Wenn sich Studenten in ihren Arbeiten mit Fachtexten auseinandersetzen, so steht zunächst die Reproduktion des Gelesenen im Vordergrund (...). Erst nach mehreren Semestern und Hausarbeiten beginnen sie zu begreifen, dass die Wissenschaftskul- tur eine eristische Kultur ist, in der publiziertes Wissen grundsätzlich kritisch zu prüfen ist. In der Folge beginnen die Studenten das Gelesene stärker zu reflektieren und zu hinterfragen. Erst relativ spät schlägt sich dies, und das auch nur bei einem Teil der Schreiber, in den Hausarbeiten nieder.« (Steinhoff 2007: 361)

Eine gezielte und systematische Unterstützung von Studierenden beim Erwerb wissenschaftlicher Bewertungskompetenz gehört - wie die Ergebnisse von Steinhoff (2007: 361ff) deutlich machen - zu den zentralen Aufgaben einer Didaktik der Wissenschaftssprache. Unter den Bedingungen des Bachelor- und Masterstudiums, das zeitlich einen sehr viel enger begrenzten Rahmen setzt als die herkömmlichen Magisterstudiengänge, stellt sich diese Notwendigkeit in noch höherem Maße.

Zentrale Fragen für die Auswertung der studentischen Rezensionen aus dem Modul sind für uns dementsprechend, inwieweit und in welchem Umfang in ihnen explizite (positive und negative) Bewertungen vorgenommen und welche Bewertungskriterien von den Studierenden herangezogen werden. Darüber hinaus stellt sich die Frage nach der inhaltlichen und sprachlichen Qualität der vorgenommenen Bewertungen und ihrer jeweiligen Begründung bzw. die Frage, 
inwieweit aus dem Korpus spezifische Problempunkte in Bezug auf das Bewerten bestimmt werden können.

Eine erste quantitative Auswertung des Korpus ergab, dass ausnahmslos in allen Rezensionen - zum größten Teil in erheblichem Umfang - Bewertungen vorgenommen werden. In sechs der Rezensionen liegt die Anzahl der Belegstellen für sprachliche Bewertungshandlungen zwischen 5 und 9, in zwölf der Rezensionen zwischen 10 und 19, zwei der Rezensionen enthalten zwischen 22 und 30 Belege. Dabei beschränken sich die Belege in den meisten Fällen nicht nur auf den Schlussteil der Rezensionen, der eine abschließende Bewertung von der Textart her zwingend vorschreibt. Vielmehr erfolgt auch eine - wenn auch in Umfang und Qualität sehr unterschiedliche - Bewertung von Einzelaspekten der rezensierten Primärtexte im Hauptteil von insgesamt 17 der ausgewerteten Rezensionen.

Im Folgenden sollen Einzelbeispiele aus dem Korpus näher untersucht werden. Dabei differenzieren wir zwischen Bewertungen, die sich auf die Qualität der Darlegung von wissenschaftlichem Wissen in den rezensierten Publikationen beziehen, und Bewertungen, die die wissenschaftliche Qualität des in der Publikation präsentierten Wissens selbst betreffen.

\subsubsection{Bewertung der Qualität der Darlegung des wissenschaftlichen Wissens}

Wissenschaftliche Rezensionen dienen im Wissenschaftsbetrieb primär der Einschätzung der Qualität des dargelegten Wissens. Allerdings ist das Kriterienraster von Rezensenten sehr viel weiter und variiert in Abhängigkeit von der Rezensionsvorlage, den eigenen Leseerwartungen des Rezensenten, dem Zielpublikum des Rezensionsorgans usw. Wer etwa eine Einführung in die Linguistik rezensiert, kann auch pragmatische Kriterien wie die Handhabbarkeit des Registers oder die Angabe von weiterführenden Lesehinweisen berücksichtigen oder auch ästhetische Kriterien wie die Übersichtlichkeit und grafische Umsetzung der Darstellung. In Rezensionen, die sich nicht nur an die Wissenschaftsgemeinschaft, sondern auch an fachlich ausgebildete Praktiker wenden, kann die Frage nach der Praxisrelevanz der Publikation eine wichtige Rolle spielen. Die Varianz der Bewertungskriterien ist auch Ausdruck der starken Leserorientierung, die die Textart Rezension aufweist. So finden sich - wie die bisherige Forschung zur Textart belegt - in Expertenrezensionen in der Regel immer auch Bewertungen zum Aufbau und der äußeren Form oder auch zum sprachlichen Stil der rezensierten Publikationen, die unter dem Stichwort der Leserfreundlichkeit zusammengefasst werden können.

Dass auch Aspekte, die nicht unmittelbar die wissenschaftliche Qualität des präsentierten Wissens betreffen, einer Bewertung unterzogen werden können und sollen, ist den Studierenden aus der exemplarischen Analyse von Expertenrezensionen durchaus bewusst. Bewertungen der Leserfreundlichkeit unter 
dem Aspekt der Strukturierung bzw. der Orientierungsfunktion zusammenfassender Textteile, die sich in ähnlicher Weise auch in Expertenrezensionen finden, werden z. B. in den folgenden Belegstellen aus unserem Korpus vorgenommen:

B6: Der Band ist klar strukturiert und in eine Einleitung und drei Kapitel gegliedert. (KB)

B7: Am Ende der ersten vier Kapitel befindet sich jeweils ein Fazit, was dem Leser eine bessere Orientierung erlaubt. (HEN)

B8: Die Strukturierung, logische Reihenfolge der Präsentierung der Gedanken und die Sprache des Textes sind leserfreundlich gehalten, so dass der Leser keine Schwierigkeiten dabei haben wird. (SAR)

Während die positive Einschätzung des benannten Aspekts der Strukturierung in Beispiel 6 ausschließlich über die Qualifizierung als klar kommuniziert wird, wird in den Beispielen 7 und 8 die positive Konsequenz der benannten Aspekte für die Rezeption explizit benannt. In Beispiel 8 erfolgt dies neben der Qualifizierung mittels des Adjektivs leserfreundlich zusätzlich durch einen Konsekutivsatz, der darauf angelegt ist, die Leserfreundlichkeit weiter zu spezifizieren, der sich jedoch durch den deiktischen Rückverweis mittels des zusammengesetzten Verweiswortes dabei (dessen präpositionaler Teil zudem falsch gewählt wurde) auf den Satzanfang als zirkulär bzw. in Bezug auf den Informationsgehalt als redundant erweist.

Eher ungewöhnlich für Expertenrezensionen (wenn auch nicht völlig ausgeschlossen) dürfte dagegen die Bewertung von Schriftgröße und Papierqualität sein:

B9: Was das Format angeht, sind die lesefreundliche Schriftgröße von ca. 10 Punkten und der Inhalt der DVD hervorzuheben, auf der sechs Ausschnitte von Kurz- und Spielfilmen angeboten werden. Nicht so sinnvoll ist allerdings die Verwendung von Glanzpapier, das das Lesen des Buches unter bestimmten Umständen erschwert. (KB)

Auffällig ist, dass die Bewertung der sprachlichen Einfachheit oder auch Komplexität der rezensierten Werke in den meisten studentischen Rezensionen des Korpus eine wichtige Rolle spielt. Eine Erklärung hierfür dürfte sein, dass die Studierenden sich in Bezug auf diesen Aspekt in viel selbstverständlicherer Weise als Experten verstehen, denen eine unmittelbare Bewertungskompetenz zukommt, als dies in Bezug auf die Bewertung der wissenschaftlichen Qualität der rezensierten Werke der Fall ist.

B10: Daneben ist die Arbeit durch die verständliche Sprache, die besonders Nichtmuttersprachlern entgegenkommt, und den strukturierten Aufbau gekennzeichnet. (REH)

B11: Das Werk hat weiterhin im Grund genommen vollkommen beispielhafte Darstellungen mit Kinder-Aufzeichnungen und-Bilder und verfügt über einen sehr guten und nichtkomplizierten sprachlichen Stil. (KHA) 
B12: Außerdem fasst die Verfasserin viele fachliche Informationen in sehr komprimierter Form zusammen, dass die Informationen unklar bzw. unverständlich für die Zielgruppe bleiben können, die sich nicht aus Fachleuten konstituiert. (ARO)

Für das Korpus insgesamt gilt, dass die Studierenden - wie auch in den vorliegenden Beispielen - sprachliche Einfachheit durchwegs als eine positive, sprachliche Komplexität dagegen in den meisten Fällen als eine negative Qualität einschätzen. Dies erscheint dort fraglos berechtigt, wo Einführungstexte rezensiert werden (was in fünf der studentischen Rezensionen der Fall ist). Nicht in allen diesen Rezensionen wird das Bewertungskriterium der sprachlichen Einfachheit des Primärtextes jedoch explizit auf die rezensierte Textart und deren Zielgruppe oder auch (wie in Beispiel 10) auf eine mögliche Zielgruppe der Rezension bezogen. Einen interessanten Fall stellt unter diesem Gesichtspunkt Beispiel 12 dar: So wird die negative Bewertung der komprimierten Darstellungsweise des Primärtextes zwar mit der Zielgruppe begründet - dabei liegt jedoch eine eklatante Fehleinschätzung in Bezug auf diese zugrunde, da es sich bei dem rezensierten Werk um eine Dissertation handelt, die sich als solche an die Wissenschaftsgemeinschaft wendet.

Während unter dem Kriterium der Leserfreundlichkeit keine Aussagen über die wissenschaftliche Qualität der rezensierten Publikationen getroffen werden, gilt dies für andere Aspekte der Qualität der Darlegung des wissenschaftlichen Wissens wie zum Beispiel die Vollständigkeit bzw. Detailliertheit (Beispiele 13, 14) und Differenziertheit (Beispiel 15) oder auch die Nachvollziehbarkeit (Beispiel 16) und Begriffsgenauigkeit (Beispiel 17) in der Darstellung durchaus:

B13: Der Verdienst des Buches liegt darin, dass es einen umfassenden Überblick über das Konzept der »rezeptionsästhetischen Literaturdidaktik « gibt. (HEN)

B14: Einige Themen werden jedoch nur mit einem einleitenden und oberflächlichen Charakter behandelt. (GIO)

B15: Auf die Themen Lesen und Schreiben der Kinder gehen Noll und Schieder im vierten Unterkapitel (61-81) ganz tief und vielseitig ein, in dem sie sich mit verschiedenen Fragen auseinandersetzen, die häufig auftauchen könnten, wenn man diese beiden Fertigkeiten, nämlich das Lesen und Schreiben, bei den Kindern beobachten und behandeln möchte. (KHA)

B16: Dieses Buch hinterlässt einen überzeugenden Eindruck beim Leser, weil die Verfasserin ihr Thema bzw. ihre Zielsetzung theoretisch und empirisch nachvollziehbar dargestellt hat. (SHE)

B17: Die dargestellten Thesen werden von Maijala gut begründet und die angewandten Begriffe werden oft klar definiert. (RAS)

Auch wenn Bewertungen dieser Art sich auf die wissenschaftliche Qualität der rezensierten Publikationen beziehen, so handelt es sich hierbei doch tendenziell um Standardbewertungen, die auch von Rezensenten, deren Fähigkeit zum fachspezifischen wissenschaftlichen Einschätzen noch nicht voll ausgebildet ist 
(vgl. Redder 2002), relativ leicht vorgenommen werden können. Sie setzen keine vollständige Durchdringung und Einordnung des in der Publikation präsentierten Wissens voraus und erfordern keine fachwissenschaftlichen Begründungen. Zudem können studentische Rezensenten hier auch auf ihre eigene Schreiberfahrung zurückgreifen: Jeder Studierende weiß wohl, dass ein längerer Text »klar gegliedert « sein soll. Studentischen Rezensionen, in denen primär die Qualität des wissenschaftlichen Wissens eingeschätzt wird, dürfte dementsprechend eine Wissenskonstellation zugrunde liegen, in der - nach der Differenzierung von Redder (2002: 21ff.) - ein forschendes Lern-Wissen bereits aufgebaut ist, jedoch gegenüber dem lernenden Forschungswissen, das die Grundlage für ein fachspezifisch fundiertes wissenschaftliches Einschätzen bildet, noch überwiegt. Die kompetente Einschätzung von wissenschaftsrelevanten Aspekten der Darlegung wissenschaftlichen Wissens kann dabei jedoch durchaus als ein wichtiger Schritt für den Erwerb einer umfassenden und differenzierten fachwissenschaftlichen Bewertungskompetenz verstanden werden.

\subsubsection{Bewertung der wissenschaftlichen Qualität des präsentierten Wissens}

Bemerkenswert ist, dass ausnahmslos alle studentischen Rezensionen des Korpus sich zumindest ansatzweise der Herausforderung stellen, die wissenschaftliche Qualität des im Primärtext präsentierten Wissens einzuschätzen - wenn auch in sehr unterschiedlichem Umfang (die Zahl der Belegstellen schwankt hier zwischen 1 und 10) und in sehr unterschiedlicher Qualität, die sich an der sachlichen Adäquatheit der Bewertung, der Realisierung einer Begründung und deren Stichhaltigkeit, aber auch an der Angemessenheit der sprachlichen Realisierung messen lässt.

Der von den Studierenden dabei mit Abstand am häufigsten als bewertungsrelevant eingestufte Aspekt ist die Stellung der Publikation im Forschungszusammenhang. Eine differenzierte, sachlich und sprachlich angemessene Einordnung gelingt in den folgenden drei Beispielen aus einer studentischen Rezension des Korpus. So verortet die Rezensentin das Werk mit dem einleitenden Satz in der Forschungslandschaft und erkennt dessen Innovationsgehalt:

B18: Die Diskussion um den didaktischen Stellenwert der Integration von Filmen in den Unterricht ist relativ aktuell. Im vorliegenden Buch wird erstmals ein klarer didaktischer Vorschlag für einen medienreflexiven Deutschunterricht angeboten. (KB)

Der eingangs zunächst allgemein benannte Innovationsgehalt wird dann im weiteren Verlauf der Rezension in Bezug auf einzelne Kapitel spezifiziert:

B19: Die Hauptleistung dieses klar strukturierten Kapitels besteht vor allem in den deskriptiven und fundierten theoretischen Überlegungen zur Didaktisierung von wichtigen filmischen Elementen, die bisher in der wissenschaftlichen Literatur der Filmdidaktik kaum eine Rolle gespielt haben. (KB) 
Auch bei Teilaspekten hebt sie Neuerungen hervor, deren spezifische Relevanz detailliert begründet wird:

B20: Ganz innovativ sind die Übungen, die mit Filmplakaten und Trailern vorgeschlagen werden. Das ist besonders interessant vor allem in Kontexten, wo der Zugang zu deutschsprachigen Filmen nicht immer einfach ist. Der Trailer als Impuls für assoziatives Schreiben, die Präsentation des Trailers ohne Ton und damit die Verfassung von Texten, oder die Erstellung eines eigenen Trailers zählen zu den Aufgaben, die dieses Element für den medienreflexiven Unterricht bieten kann. (KB)

Solche detaillierten Begründungen fehlen in anderen Arbeiten z. T. vollständig - so in dem folgenden Beispiel, in dem die Relevanz des Beitrags für den Forschungszusammenhang zwar behauptet, jedoch an keiner Stelle der Rezension (z. B. durch einen Vergleich mit anderen Forschungsarbeiten oder durch weitere Ausführungen zur spezifischen Leistung des Beitrags) weiter belegt wird:

B21: Der vorliegende Artikel zählt zu den wichtigsten Beiträgen über das Lesen und den Leseprozess. Er beschäftigt sich mit dem Thema »Lesen in L2 als Leser Text Interaktion «. (SAR)

Eine stichhaltige Begründung fehlt auch im folgenden Beispiel, auch wenn in ihm die als innovativ qualifizierten Kriterien zunächst in Form einer Aufzählung benannt werden. So wird die zunächst eindeutig vorgenommene Qualifizierung als innovativ bereits im ersten Satz durch die Vagheit der Formulierung insgesamt einen überzeugenden Eindruck hinterlassen wieder abgeschwächt und im letzten Satz durch die Einengung auf eine Selbsteinschätzung des Autors als Beleg für die Relevanz der Kriterien wieder in Frage gestellt:

B22: Dieses Kapitel umfasst einige Kriterien, die innovativ sind und insgesamt einen überzeugenden Eindruck hinterlassen. Die Kriterien sind Aktualität, positive Konnotation oder Provokation, Altersgemäßheit, inhaltliche und sprachliche Angemessenheit, interkulturelle Perspektive, Vergrößerung des Wahrnehmungsfeldes und Freude an der Lektüre. Dies sind überaus nach der Ansicht Koppensteiners wichtige Kriterien für jeden, der sich mit Literatur im Sprachenunterricht beschäftigt. (SAL)

Ein weiterer Aspekt der Bewertung der Stellung der rezensierten Werke, der in einer Reihe von Rezensionen hervorgehoben wird, ist deren Relevanz für Anschlussforschungen. So auch im folgenden Beispiel aus einer Rezension zu einem Sammelband: Zum einen wird die Repräsentativität des Bandes für den aktuellen Forschungsstand betont und mit der Anerkanntheit der Autoren in der fachwissenschaftlichen Gemeinschaft begründet, zum anderen wird unter Einsatz der Kollokation den Weg bahnen ${ }^{1}$ auf die Perspektiven für weitere Forschungen hingewiesen:

${ }^{1}$ Die Rezensentin bedient sich hier der in der allgemeinen Wissenschaftssprache höchst frequenten Weg-Metapher. Zum Bildbereich des Weges in Bezug auf Sprachhandlungsverben in der alltäglichen Wissenschaftssprache vgl. Fandrych (2004). 
B23: Als eine Sammlung der Beiträge von bedeutsamen und anerkannten Autoren im Rahmen der Fremdsprachendidaktik und Dramapädagogik fasst dieser Band das aktuelle Panorama über den Forschungsstand zur Inszenierung im Fremdsprachenunterricht zusammen und bahnt damit den Weg für neue Perspektiven. (YL)

Um welche Perspektiven es sich im Einzelnen handelt, wird von der Studentin dann in den sich anschließenden Ausführungen zu den einzelnen Beiträgen detailliert ausgeführt.

Im Gegensatz dazu bleibt die Bewertung in dem folgenden Ausschnitt aus einer weiteren Rezension insgesamt sehr allgemein und inhaltlich unspezifisch. Zudem zeigt der Ausschnitt in charakteristischer Weise die bei einigen Studierenden bestehenden Probleme in der Einschätzung der Frage, welche Aspekte des Primärtextes bewertungswürdig sind bzw. welche Aspekte als wissenschaftlich selbstverständlich vorauszusetzen sind und damit keiner expliziten positiven Bewertung bedürfen. Es handelt sich um einen Abschnitt, der dem abschließenden Teil einer Rezension entnommen ist und in dem versucht wird, die Vorzüge und Nachteile des rezensierten Werkes zusammenzufassen:

B24: Anhand der empirischen Untersuchung gelangt die Verfasserin zu der Einschätzung des Abschlussprojekts. Die zahlreichen Beispiele für die Integration der Rock- und Popmusik im Lyrikunterricht besitzen einen hohen Stellenwert, weil sie dem Leser verschiedene Auffassungen über dieses Thema darbieten. Einen besonderen Stellenwert gewinnt das Buch durch den Bezug auf Autor/innen aus anderen Fachgebieten, weil die Verfasserin ihre Aussagen und Untersuchungen damit auf eine breite Basis stellt. Die Forschungsfragen, die die Verfasserin vorgeschlagen hat, sind überaus relevant, da sie weitere Diskussionen und Forschungen herausfordern. (SHI)

Dass die rezensierte Autorin den Forschungsstand aufarbeitet (verschiedene Auffassungen über das Thema darbieten) und Quellen aus anderen für das Thema relevanten Fachgebieten zitiert (Bezug auf Autor/innen aus anderen Fachgebieten) ist grundlegender Bestandteil wissenschaftlichen Arbeitens und damit schwerlich als besondere Leistung der Autorin herauszustellen. Hier hätte wesentlich konkreter herausgearbeitet werden können, welchen Nutzen das Werk tatsächlich für den Forschungsbereich mit sich bringt. Ein grundlegendes Missverständnis liegt zudem in Bezug auf den Begriff Forschungsfrage vor, die hier als Herausforderung für weitere Forschungen hervorgehoben wird, anstatt im Einzelnen nach der Qualität ihrer Beantwortung in der Publikation zu fragen.

Eine Fehleinschätzung des Publikationsziels liegt im folgenden Beispiel aus einer Rezension zu einer Arbeit über die Verbvalenz im Deutschen und Arabischen vor, deren Qualität in ihrem Beitrag für den Sprachvergleich zu suchen wäre und nicht in der Klärung von Grundbegriffen der Valenztheorie, in denen sich der Autor auf andere valenztheoretische Grundlagenwerke bezieht:

B25: Mit seiner detailreichen Untersuchung leistet der Vf. zweifellos einen wichtigen Beitrag zur Darstellung der Grundbegriffe der Valenztheorie (...). (MOH) 
Die bisher aufgeführten Beispiele machen einige charakteristische Herausforderungen und Schwierigkeiten bei der Formulierung positiver Kritik deutlich. Demgegenüber stellt die Äußerung negativer Einschätzungen für die Studierenden eine noch größere Herausforderung dar: Zum einen, da dies bedeutet, sich als »Novize« in der Wissenschaftsgemeinschaft gegenüber der Arbeit eines »Experten « (Steinhoff 2007: 362) negativ-kritisch zu positionieren; zum anderen, da die Äußerung negativer Kritik neben einer überzeugenden Begründung einen hohen Grad der Differenzierung in der Darstellung erfordert. Dies zeigt auch der Blick auf Expertenrezensionen, für die hier zumindest ein Beispiel zitiert werden soll:

B26: Dies deutet zumindest darauf hin, dass der kontrastive Ansatz, der vor allem in der Interpretation der Daten (...) noch dominiert und der ja von einer weitgehend unreflektierten Übertragung eigenkultureller Standards in interkulturelle Situationen ausgeht, offenbar falsch oder zumindest doch sehr einseitig ist. Hier hätten sich Forschungsperspektiven in eine stärker interaktionistische Richtung ergeben können, die die stagnierende Diskussion über das interkulturelle Paradigma hätten beleben und das festgefahrene Interkulturalitätsparadigma hätten aufbrechen können. (Altmayer 2009: 58)

Auffällig sind hier einschränkende bzw. abschwächende Ausdrücke wie darauf hindeuten (statt z. B. zeigen), zumindest und weitgehend sowie stark hörerbezogene Ausdrücke wie ja, doch und offenbar, die an die Zustimmung des Hörers appellieren, sowie die abschwächende alternative Nennung von einseitig gegenüber dem zunächst als Bewertung genannten falsch. Sie können zum Teil als Höflichkeitsmarker, zum Teil aber auch als Ausdruck für eine gewisse Vorsicht gegenüber einem möglichen Absolutheitsanspruch verstanden werden. ${ }^{1}$ Interessant erscheint uns an dem Beispiel darüber hinaus, dass hier offensichtlich der klaren Formulierung einer alternativen Perspektive, wie sie im zweiten Teil des Beispiels formuliert ist, eine wichtige Funktion für die Begründung der Kritik zukommt. Trotz der hohen Anforderungen erproben sich immerhin 12 der 20 Verfasser der von uns untersuchten studentischen Rezensionen, also $60 \%$, an einer kritischen Einschätzung des von ihnen rezensierten Primärtextes. Auch wenn die Äußerung von negativ-kritischen Einschätzungen längst nicht in allen Fällen überzeugend gelingt, so finden sich im Korpus doch auch eine Reihe von Belegstellen, in denen die Kritik eine intensive fachliche Auseinandersetzung mit dem rezensierten Text und eine souveräne Handhabung wissenschaftlicher Bewertungskriterien erkennen lässt und auch an der sprachlichen Oberfläche differenziert formuliert und begründet wird. Auffällig ist, dass das Bewertungskriterium der Richtigkeit, das

1 Ausdrücke wie diese werden in der Wissenschaftssprachforschung zumeist als Heckenausdrücke klassifiziert, denen die Funktion zugeschrieben wird, das »Gesicht « des Autors der rezensierten Publikation wie auch des Autors der Rezension zu wahren (so auch bei Steinhoff 2007: 376). Demgegenüber plädiert Graefen (2000) für eine differenzierte Interpretation der Einzelbeispiele. 
in dem von Steinhoff untersuchten Expertenkorpus am häufigsten als Referenz für die Äußerung von Kritik belegt ist (vgl. Steinhoff 2007: 364), in den untersuchten Rezensionen in keinem Fall zum Einsatz kommt. In Übereinstimmung mit den Ergebnissen von Steinhoff (2007: 372ff.) wird in den hier untersuchten Rezensionen negative Kritik meist nicht direkt geübt, sondern abgemildert. Insgesamt tendieren die studentischen Rezensionen in der Sache jedoch zu einer weit vorsichtigeren Kritik als dies z. B. auch in der oben zitierten Expertenrezension der Fall ist, in der - ebenfalls in sprachlich abgemilderter Form - das Kriterium der Richtigkeit zum Einsatz kommt.

Dabei wird auch die von Steinhoff (2007: 375f.) beschriebene Strategie verwendet, zunächst auf positive Aspekte eines Textes hinzuweisen, um anschließend durch eine »Wendung « auf Defizite aufmerksam zu machen:
B27: Anhand eines relativ großen Korpus (...) ist dem Autor eine detaillierte Untersuchung sprachlicher formeller, und funktioneller Aspekte der Titel von wissenschaftlichen Arbeiten gelungen, die einen sehr wichtigen Beitrag zur Erforschung von Titeln darstellt.
Nachteile hat allerdings die in der vorliegenden Arbeit verwendete Betrachtungsweise der Titel als Stellvertreter, d.h. die Untersuchung der Titel getrennt von ihren zugehörigen Texten. Wichtige semantische Merkmale, wie beispielsweise metaphorische Bilder in Titeln, kann diese Betrachtungsweise kaum erfassen. $(\mathrm{AH})$
B28: Eine genaue Beobachtung eines in sechs Einheitsstunden geteilten Englischunterrichts wird durchgeführt, wobei zu kritisieren ist, dass es nicht deutlich wird, ob die Autorin die Rolle der Kursleiterin oder der Beobachterin übernimmt. Insofern wird auch nicht darauf eingegangen, ob und in welchem Maße diese Beobachtung die zu analysierende Situation beeinflusst hat. (AID)

In beiden Beispielen werden durch die sprachliche Verflechtung von positiven und negativen Aspekten differenzierte Bewertungen der wissenschaftlichen Qualität der Untersuchungen vorgenommen: Gewürdigt wird zunächst die Untersuchung als Ganze und der mit ihr verbundene Erkenntnisgewinn (Beispiel 27) bzw. das Untersuchungsdesign als solches (Beispiel 28). In einem zweiten Schritt wird die grundsätzlich positive Bewertung mittels des Adverbs allerdings bzw. mittels des nebensatzeinleitenden wobei in Bezug auf den im Folgenden näher ausgeführten Aspekt eingeschränkt. ${ }^{1}$ In einem dritten Schritt erfolgt dann eine Begründung der Kritik, indem die Konsequenzen, die sich aus der in Beispiel 27 kritisierten Einschränkung der Untersuchungsperspektive (diese Betrachtungsweise) bzw. aus der in Beispiel 28 kritisierten mangelnden Offenlegung des Forschungsdesigns (wird nicht deutlich; wird nicht darauf eingegangen) ergeben, verbalisiert werden. Eine Kritikabschwächung erfolgt dabei in Beispiel 28 ausschließlich über die unmittelbare Verknüpfung von positiven und negativen Aspekten, während in

1 Weitere sprachliche Mittel, die im Zusammenhang einer differenzierten Formulierung von Kritik eingesetzt werden, sind zwar...aber und jedoch. 
Beispiel 27 mit der Benennung des kritisierten Aspekts als Nachteil (wodurch das gleichzeitige Vorhandensein von Vorteilen der gewählten Untersuchungsperspektive impliziert ist) und dem Einsatz des Adverbs kaum weitere charakteristische Sprachmittel zur Abschwächung von Kritik verwendet werden. Der Anspruch auf wissenschaftliche Objektivität der Einschätzungen kommt dabei sprachlich in beiden Beispielen in der Wahl von unpersönlichen Verbkonstruktionen zum Ausdruck (Nachteile hat...; werden kaum erfasst; ist zu kritisieren).

Eine wissenschaftssprachlich differenzierte Verbalisierung wie in den Beispielen 27 und 28 gelingt dabei längst nicht in allen studentischen Rezensionen. Einige charakteristische Schwierigkeiten bzw. Problempunkte im Zusammenhang der Formulierung insbesondere von negativer Kritik sollen an den folgenden sechs Belegstellen aus dem Korpus deutlich gemacht werden:

B29: Viele seiner Beispiele aber handeln von den Navajoindianern oder der arabischen Welt im Allgemeinen. Da ich der Meinung bin, dass es in der arabischen Welt noch viele unterschiedlichen Kulturen gibt und dass nicht alle Araber dieselbe Verhaltensmuster zeigen, finde ich es schade, dass ein Anthropologe auf dergleichen Weise verallgemeinert. (VAN)

Zwar wird in Beispiel 29 eine wissenschaftsmethodisch und der rezensierten Publikation angemessene Kritik formuliert. Anders als in den vorhergehenden Beispielen wird diese jedoch durch den Einsatz von Formulierungsroutinen wie da ich der Meinung bin und finde ich es schade, die ihren Ort in alltagssprachlichen Argumentationszusammenhängen haben, als eine Kritik verbalisiert, die sich aus der persönlichen Perspektive der Rezensentin herleitet und damit explizit nicht auf eine fachliche Argumentation gegründet ist. Während die Formulierungen in Beispiel 29 auf mangelnde Erfahrung mit der Wissenschaftssprache zurückzuführen sein dürfte, liegt der Fall in Beispiel 30 noch einmal anders:

B30: Das Werk hat weiterhin im Grund genommen vollkommen beispielhafte Darstellungen mit Kinder-Aufzeichnungen und -Bilder und verfügt über einen sehr guten und nichtkomplizierten sprachlichen Stil. Es ist leider bloß - meines Erachtens - nicht so genug theoretisch fundiert, und zwar dass man sich bei der Darstellung kaum theoretische Überlegungen finden, was mir doch bloß aufgefallen ist. (KHA)

Auch in dieser Rezension wird mit der mangelnden theoretischen Fundierung eine durchaus begründete wissenschaftliche Kritik formuliert, wobei - ähnlich wie in Beispiel 27 und 28, wenn auch (wissenschafts)sprachlich weit weniger versiert $^{1}$ - eine differenzierte Beurteilung über die Verknüpfung von aufeinander bezogenen positiven und negativen Aspekten angestrebt wird. Darüber hinaus wird die Verbalisierung der negativen Kritik mit dem in Rezensionen an dieser

1 Stilistisch bewegen sich die Formulierungen im vorliegenden Beispiel im Bereich mündlicher Sprachverwendung und zeigen, dass die Sprachkompetenz des Verfassers im Bereich der geschriebenen Wissenschaftssprache noch erhebliche Defizite aufweist. 
Stelle charakteristischen Adverb leider eingeleitet. Allerdings wird die Geltung der Kritik durch die Formulierungen meines Erachtens und was mir doch bloß aufgefallen ist auf die Perspektive des Rezensenten zurückgenommen, die hier explizit als eingeschränkte Perspektive verbalisiert ist (doch bloß). Ähnliche Beispiele finden sich auch im Korpus von Steinhoff (2007). Der Einsatz solcher »Unsicherheitsmarker « könnte darauf zurückzuführen sein, dass Studierende sich z. T. gegenüber der »Autorität der publizierten Texte« (Kruse/Ruhmann 1999, zitiert nach Steinhoff 2007: 380) nur eine eingeschränkte Kritikkompetenz zugestehen. Es ist anzunehmen, dass diese Schwierigkeiten bei ausländischen Studierenden in noch stärkerem Maße auftreten, zum einen wegen der fehlenden muttersprachlichen Kompetenz, zum anderen weil in verschiedenen Wissenschaftstraditionen der Respekt vor dem autoritativen geschriebenen Text unterschiedlich groß ist.

Eine weitere charakteristische Problematik, für die sich in unserem Korpus mehrere Beispiele finden, zeigt die folgende Belegstelle:

B31: Die Verfasser haben sich im Buch auf den literarischen Text bzw. auf das Lesen von literarischen Texten konzentriert. Leider haben sie nur schriftliche Texte berücksichtigt. Interessant/wünschenswert wäre auch eine Auseinandersetzung mit der Rolle und dem Potenzial von Hörtexten im FSU. (HEN)

Zwar bedient sich die Rezensentin in diesem Beispiel mit dem kritikeinleitenden Einsatz des Adverbs leider und der positiven Verbalisierung des fehlenden Aspekts in einem durch wünschenswert eingeleiteten Konjunktiv-Satz charakteristischer Formulierungsroutinen im Zusammenhang von Rezensionen. Ähnlich wie in Beispiel 25 liegt hier jedoch eine Fehleinschätzung der Zielsetzung der Publikation vor, da das Fehlen eines Aspekts kritisiert wird, der außerhalb der Fragestellung der Publikation liegt. Eine solche Fehleinschätzung könnte darauf beruhen, dass hier ein Aspekt vermisst wird, der im eigenen Forschungsinteresse der Rezensentin liegt.

Schwierigkeiten bereitet manchen Studierenden auch die angemessene Integration und argumentative Einbindung von negativer Kritik in den Gesamttext, wie zum Beispiel in der folgenden Belegstelle, die am Schluss einer Rezension steht, in der im Gesamttext kontinuierlich und ausschließlich mittels Adjektiven eine positive Einschätzung des rezensierten Werkes verbalisiert wird:

B32: Die Assoziation von den verschiedenen Methoden der Literaturdidaktik mit den jeweiligen Schulebenen sind eine sehr interessante und gründliche Information für den Leser, da er, was er für seinen Unterricht wichtig findet, leicht erkennen und verwenden kann.

Unter Umständen könnte der Leser subjektive Argumente seitens der Autorin sowie praktische Vorstellungen der Theorie vermissen. (LUC)

Die Verbalisierung einer negativen Kritik im Schlusssatz der Rezension (zumal ohne dass diese an einer anderen Stelle des Textes eine Entsprechung fände) deutet darauf hin, dass die Rezensentin davon ausgeht, dass negative Kritik notwendiger Bestandteil der Textart Rezension ist. Der Eindruck, dass diese hier 
ausschließlich im Sinne einer »Pflichtübung « realisiert wird, wird dabei durch den Einsatz des Konjunktiv II sowie die adverbiale Bestimmung unter Umständen, die die Gesamtäußerung in den Modus der Unbestimmtheit setzt, noch verstärkt. Dass hier darüber hinaus auch noch grundlegende (Formulierungs-)Schwierigkeiten in Bezug auf die Einschätzung der wissenschaftlichen Qualität von Publikationen bestehen, macht die weitgehend unverständliche Formulierung deutlich, das Werk lasse praktische Vorstellungen der Theorie vermissen.

Die Auswertung des Korpus in Hinblick auf die vorgenommenen Bewertungshandlungen zeigt $\mathrm{u}$. E. deutlich die Leistungsfähigkeit der Textart Rezension für die systematische Einübung des wissenschaftlichen Bewertens. Die Analyse der Belegstellen verdeutlicht darüber hinaus, dass die für Rezensionen charakteristischen Bewertungshandlungen durchaus unterschiedliche Komplexitätsgrade aufweisen und an die Studierenden dementsprechend auch unterschiedlich hohe Anforderungen stellen: Diese reichen von den relativ einfachen sprachlichen Realisierungsformen im Zusammenhang des Bewertens von Elementen der äußeren Form, die sich - weitgehend unabhängig von der wissenschaftlichen Qualität der Publikation - auf Aspekte der Leserfreundlichkeit der Publikation beziehen, über die Einschätzung und Bewertung von wissenschaftsrelevanten Aspekten der Darlegung wissenschaftlichen Wissens bis zu komplexen Formen einer differenzierten Beurteilung der wissenschaftlichen Qualität des in der rezensierten Publikation präsentierten Wissens. Aus didaktischer Sicht empfiehlt es sich deshalb in Kursen zur Wissenschaftssprache, charakteristische sprachliche Mittel und feste Routineformeln des Bewertens in Rezensionen zunächst im Zusammenhang der Bewertung der Qualität der Darlegung des wissenschaftlichen Wissens einzuüben, zumal hier auch bei fachwissenschaftlich noch weniger versierten Studierenden mit einer selbstverständlicheren Kompetenz in der Einschätzung von deren Qualität gerechnet werden kann. Für die systematische Einübung komplexerer Bewertungshandlungen bietet sich dagegen die systematische Arbeit mit authentischen Beispielen aus studentischen Rezensionen an (siehe hierzu auch Fischer/Moll 2002: 44ff.), die gemeinsam auf ihre Stärken und Defizite hin analysiert und überarbeitet werden können.

\section{Literatur}

Dallmann, Sabine: »Die Rezension: Zur Charakterisierung von Texttyp, Darstellungsart und Stil«. In: Fleischer, Wolfgang (Hrsg.): Sprachnormen, Stil und Sprachkultur. Berlin: Zentralinstitut für Sprachwissenschaft der Akademie der Wissenschaften der DDR, 1979, 58-97.

Dalmas, Martine: »Der Weisheit letzter Schluss ... Zur Funktion des Schlusswortes in Rezensionen«. In: Jakobs, Eva-Maria; Rothkegel, Annely (Hrsg.): Perspektiven auf Stil. Tübingen: Niemeyer, 2001a, 305-319. 
Dalmas, Martine: »Empfehlen und Ablehnen in wissenschaftlichen Rezensionen: Versuch eines deutsch-französischen Vergleichs«. In: Wotjak, Gerd (Hrsg.): Studien zum romanischdeutschen und innerromanischen Sprachvergleich. Frankfurt a. M.: Lang, 2001b, 467-477.

Ehlich, Konrad: »Zur Analyse der Textart `Exzerpt««. In: Frier, Wolfgang (Hrsg.): Pragmatik - Theorie und Praxis. Amsterdam: Rodopi, 1981, 379-401.

Ehlich, Konrad: »Deutsch als fremde Wissenschaftssprache«, Jahrbuch Deutsch als Fremdsprache 19 (1993), 13-42.

Ehlich, Konrad: »Die Lehre der deutschen Wissenschaftssprache: sprachliche Strukturen, didaktische Desiderate«. In: Kretzenbacher, Heinz L.; Weinrich, Harald (Hrsg.): Linguistik der Wissenschaftssprache. Berlin: de Gruyter, 1995, 325-351.

Ehlich, Konrad: »Universitäre Textarten, universitäre Struktur«. In: Ehlich, Konrad; Steets, Angelika (Hrsg.) 2003, 13-28.

Ehlich, Konrad; Graefen, Gabriele: „Sprachliches Handeln als Medium diskursiven Denkens: Überlegungen zur sukkursiven Einübung in die deutsche Wissenschaftskommunikation«, Jahrbuch Deutsch als Fremdsprache. Band 27 (2001), 351-378.

Ehlich, Konrad; Steets, Angelika (Hrsg.): Wissenschaftlich schreiben - lehren und lernen. Berlin: de Gruyter, 2003.

Fandrych, Christian: »Herausarbeiten vs. Illustrate: Kontraste bei der Versprachlichung von Sprechhandlungen in der englischen und deutschen Wissenschaftssprache «. In: Ehlich, Konrad (Hrsg.): Mehrsprachige Wissenschaft - europäische Perspektiven. 2002, 25 Seiten. http://www.euro-sprachenjahr.de/Fandrych.pdf (06.07.12).

Fandrych, Christian: »Bilder vom wissenschaftlichen Schreiben: Sprechhandlungsausdrücke im Wissenschaftsdeutschen: Linguistische und didaktische Überlegungen«. In: Wolff, Armin; Ostermann, Torsten; Chlosta, Christoph (Hrsg.): Integration durch Sprache. Regensburg: FaDaF 2004, 269-291 (MatDaF-Materialien Deutsch als Fremdsprache 73).

Fandrych, Christian: »Aufgeklärte Zweisprachigkeit als Ziel und Methode der Germanistik nichtdeutschsprachiger Länder«. In: Schmölzer-Eibinger, Sabine; Weidacher, Georg (Hrsg.): Textkompetenz. Tübingen: Narr, 2007, 275-298.

Fandrych, Christian; Thurmair, Maria: Textsorten im Deutschen: Linguistische Analysen aus sprachdidaktischer Sicht. Tübingen: Stauffenburg, 2011.

Feilke, Helmuth; Lehnen, Katrin: »Wissenschaftlich referieren - Positionen wiedergeben und konstruieren «, Der Deutschunterricht: Wissenschaftliches Schreiben LXIII/5 (2011), 34-44.

Fischer, Almut; Moll, Melanie: »Der Sprachkurs >Wissenschaftssprache Deutschく: Ein Angebot speziell für ausländische Studierende«. In: Redder, Angelika (Hrsg.): »Effektiv studieren «: Texte und Diskurse in der Universität. Duisburg: OBST, 2002 (Osnabrücker Beiträge zur Sprachtheorie: Beiheft 12), 41-54.

Foschi Albert, Marina: »Kulturspezifische Züge schriftlicher Textsorten am Beispiel italienisch- und deutschsprachiger wissenschaftlicher Rezensionen «. In: Dalmas, Martine; Foschi Albert, Marina; Neuland, Eva (Hrsg.): Wissenschaftliche Textsorten im Germanistikstudium deutsch-italienisch - französisch kontrastiv: Trilaterales Forschungsprojekt in der Villa Vigoni (2007-2008). Loveno di Menaggio: Villa Vigoni, 2009, 258-277. http://www.villavigoni.it/fileadmin/user_upload/pdfs/WissenschaftlicheTextsortenGermanistikstudium.pdf (06.07.12).

Glück, Helmut (Hrsg.): Metzler-Lexikon Sprache. 3., neubearbeitete Auflage. Stuttgart: Metzler, 2005.

Graefen, Gabriele: »»Hedging als neue Kategorie? Ein Beitrag zur Diskussion«. In: Europa Universität Viadrina, Kulturwissenschaftliche Fakultät (Hrsg.): Hedging und Diskurs, 2000. http://www.kuwi.europa-uni.de/de/lehrstuhl/sw/sw2/forschung/hedging/index.html (06.07.12). 
Heine, Lena; Schramm, Karen: »Lautes Denken in der Fremdsprachenforschung: Eine Handreichung für die empirische Praxis«. In: Vollmer, H. Johannes (Hrsg.): Synergieeffekte in der Fremdsprachenforschung: Empirische Zugänge, Probleme, Ergebnisse. Frankfurt a. M.: Lang, 2007, 167-206.

Hintze, Martina: »Zur Untersuchung von Fachtextsorten, dargestellt an der wissenschaftlichen Rezension«. In: Weber, Siegfried (Hrsg.): Fachkommunikation in deutscher Sprache: Ergebnisse, Probleme und Methoden der Fachsprachenforschung. Leipzig: Verlag Enzyklopädie, 1989, 131-143.

Hutz, Matthias: »>Insgesamt muss ich leider zu einem ungünstigen Urteil kommen.< Zur Kulturspezifik wissenschaftlicher Rezensionen im Deutschen und Englischen«. In: Fix, Ulla; Habscheid, Stephan; Klein, Josef (Hrsg.): Zur Kulturspezifik von Textsorten. Tübingen: Stauffenburg, 2001, 109-130.

Kaiser, Dorothee: Wege zum wissenschaftlichen Schreiben: Eine kontrastive Untersuchung zu studentischen Texten aus Venezuela und Deutschland. Tübingen: Stauffenburg, 2002.

Kristo, Ema: »Deutsche und albanische Rezensionstexte im Vergleich«. In: Skiba, Dirk (Hrsg.): Textmuster: schulisch - universitär - kulturkontrastiv. Frankfurt a. M.: Lang, 2010, 29-46.

Liang, Yong: »Zu soziokulturellen und textstrukturellen Besonderheiten wissenschaftlicher Rezensionen: Eine kontrastive Fachtextanalyse Deutsch/Chinesisch «, Deutsche Sprache 19, 4 (1991), 289-311.

Pätzold, Jörg: Beschreibung und Erwerb von Handlungsmustern. Beispiel: Rezensionen wissenschaftlicher Publikationen. Berlin: Zentralinstitut für Sprachwissenschaft der Akademie der Wissenschaften der DDR, 1986.

Piitulainen, Marja-Leena: »Interpersonalität und Textsortenstile: kontrastive Aspekte (Deutsch-Finnisch)«. In: Jakobs, Eva-Maria; Rothkegel, Annely (Hrsg.): Perspektiven auf Stil. Tübingen: Niemeyer, 2001, 187-204.

Redder, Angelika: „Sprachliches Handeln in der Universität - das Einschätzen zum Beispiel«. In: dies. (Hrsg.): »Effektiv studieren«: Texte und Diskurse in der Universität. Duisburg: OBST, 2002 (Osnabrücker Beiträge zur Sprachtheorie, Beiheft 12), 5-28.

Rehbein, Jochen: Komplexes Handeln: Elemente zur Handlungstheorie der Sprache. Stuttgart: Metzler, 1977.

Ripfel, Martha: »Fachtextsorten der Wissenschaftssprachen II: die wissenschaftliche Rezension«. In: Hoffmann, Lothar; Kalverkämper, Hartwig; Wiegand, Herbert Ernst (Hrsg.) in Verbindung mit Christian Galinski und Werner Hüllen: Languages for Special Purposes / Fachsprachen. An International Handbook of Special-Language and Terminology Research / Ein internationales Handbuch zur Fachsprachenforschung und Terminologiewissenschaft. Teilband 1. Berlin: de Gruyter, 1997, 488-493.

Rösler, Dietmar: E-Learning: Eine kritische Einführung. 2. Aufl. Tübingen: Stauffenburg, 2007. Steinhoff, Torsten: Wissenschaftliche Textkompetenz. Sprachgebrauch und Schreibentwicklung in wissenschaftlichen Texten von Studenten und Experten. Tübingen: Niemeyer, 2007.

Stezano Cotelo, Kristin: Verarbeitung wissenschaftlichen Wissens in Seminararbeiten ausländischer Studierender: Eine empirische Sprachanalyse. München: iudicium, 2008.

Wiegand, Herbert-Ernst: »Nachdenken über wissenschaftliche Rezensionen. Anregungen zur linguistischen Erforschung einer wenig erforschten Textsorte«, Deutsche Sprache 2 (1983), 122-137.

Zillig, Werner: »Textsorte Rezension«. In: Detering, Klaus; Schmidt-Radefeldt, Jürgen; Sucharowski, Wolfgang (Hrsg.): Akten des 16. Linguistischen Kolloquiums Kiel, 1981; I: Sprache beschreiben und erklären; II: Sprache erkennen und verstehen. Tübingen: Niemeyer, 1982, 199-208. 
Verwendete Rezensionen:

Altmayer, Claus: Rezension zu Casper-Hehne, Hiltraud: Deutsch-amerikanische Alltagskommunikation. Zur Beziehungsarbeit in interkulturellen Gesprächen. Tübingen: Niemeyer, 2006, Deutsch als Fremdsprache 46, 1 (2009), 56-58.

Florin, Karl-Walter: Rezension zu Rösler, Dietmar (2007): E-Learning: Eine kritische Einführung. (2. Aufl.) Tübingen: Stauffenburg, Info DaF 35, 2/3 (2008), 326-329.

Informationen zu den Autoren:

Die Autoren lehren wissenschaftliches Schreiben, Arbeiten und Präsentieren im Modul »Deutsch als Wissenschaftssprache«, das für internationale Masterstudierende am Herder-Institut, Universität Leipzig, angeboten wird.

Albrecht Klemm

promoviert im Internationalen Promotionsstudiengang »Deutsch als Fremdsprache / Transcultural German Studies« (Leipzig - Arizona) über die erwerbsfördernde Wirkung schriftlicher Fehlerkorrektur im DaF-Unterricht. Er ist wissenschaftlicher Mitarbeiter am Herder-Institut und koordiniert den Aufbau einer Deutschabteilung an der Salahaddin Universität in Erbil (Irak).

\section{Stefan Rahn}

hat mehrere Jahre als DAAD-Lektor am King's College London sowie an der Außenstelle London des DAAD gearbeitet. Er ist wissenschaftlicher Mitarbeiter am HerderInstitut und promoviert über sprachliches Handeln und Handlungsmuster in mündlichen Hochschulprüfungen mit deutschen und internationalen Studierenden. Er forscht v. a. zur Wissenschaftssprache und ist am Projekt »Gesprochene Wissenschaftssprache kontrastiv« (GeWiss) beteiligt.

\section{$>$ Dr. Renate Riedner}

ist seit 2003 wissenschaftliche Mitarbeiterin am Herder-Institut. Sie hat 1996 am Institut für Deutsch als Fremdsprache an der Universität München promoviert und war an der Ludwig-Maximilians-Universität in München sowie als DAAD-Lektorin an Universitäten in Kairo tätig. Ihre Arbeitsschwerpunkte liegen im Bereich der Wissenschaftssprache und der Literatur- und Kulturstudien im Fach Deutsch als Fremdsprache.

Weitere Informationen unter http://www.uni-leipzig.de/herder/hi.site,postext,mitarbeiter.html?PHPSES$\mathrm{SID}=562$ cace75e6sp0t1a6rcq7v5dtk5vee9 